\title{
Attribution and identification of absorbed components by HPLC-DAD-ESI-MS after oral administration of Erhuang decoction
}

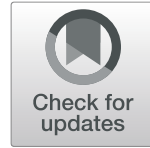

Jinglong Wang ${ }^{1 * \dagger} \mathbb{D}$, Dandan Zheng ${ }^{1 \dagger}$, Nan Xu$^{2}$, Chao Zhang ${ }^{3}$, Yingzi Wang ${ }^{4}$, Xiumei Sun ${ }^{3}$ and Zhaowang Zhang ${ }^{3}$

\begin{abstract}
To realize the attribution and identification of absorbed components in rat serum after oral administration of Erhuang decoction prepared by semi-bionic enzyme extraction method, the fingerprints of serum samples were established using a HPLC-DAD-ESI-MS method. Thirty-two peaks in Erhuang decoction and 24 peaks in rat serum after oral administration of Erhuang decoction were detected. Among the 24 peaks detected in rat serum, 25 compounds were identified by comparing the retention time and mass spectrometry data with that of reference compounds, or by mass spectrometry analysis and retrieving the reference literatures. Among the identified 25 compounds in vivo, 24 were the original form of compounds absorbed from the detected compounds in vitro, and one was the metabolite compounds of licorice. By analyzing the mass spectrometry or ultraviolet absorption characteristics, other unidentified compounds in vivo were deduced to be the endogenous metabolites in serum or the original form and metabolites of the compounds existed in vivo. Results indicated that HPLC-DAD-ESI-MS is suitable for identifying the bioactive constituents in serum after oral administration of Erhuang decoction, and the findings would be beneficial to further research and development of the pharmacodynamic substance base of Erhuang decoction.
\end{abstract}

Keywords: Erhuang decoction, Semi-bionic enzyme extraction, HPLC-DAD-ESI-MS, Bioactive constituents, Identification

\section{Introduction}

Traditional Chinese medicine (TCM) is a mixture of ingredients. Some of them effect as original form compounds, while some others can only be activated through metabolic transformation in vivo. As only the ingredients absorbed into the blood are possibly effective, the components that can migrate into the blood potentially exert the therapeutic effects. Serum pharmacochemistry provides the technical support for studying the mixture ingredients of TCM. Serum fingerprint can be used as a guidance of serum pharmacochemistry, which selects appropriate methods to enrich the components in the blood highly

\footnotetext{
* Correspondence: jlwang8121@163.com

${ }^{\dagger}$ Jinglong Wang and Dandan Zheng contributed equally to this work. ${ }^{1}$ College of Food Sciences and Pharmaceutical Engineering, Zaozhuang University, Zaozhuang 277160, China

Full list of author information is available at the end of the article
}

effectively to promote the study on pharmacodynamic material base of TCM. For example, the fingerprints of Yin Chen Hao Tang (YCHT), a traditional Chinese medical formula, were established with 21 compounds in rat plasma after oral administration of YCHT detected, and all of them were identified (Wang et al. 2008). Miao et al. developed an HPLC/DAD/ESI-MS ${ }^{\mathrm{n}}$ approach to identify the chemical components and absorbed ingredients (and metabolites) of Shenshao Tablet in rat plasma and urine (Miao et al. 2018).

Erhuang decoction is a compound prescription prepared from three kinds of Chinese herbs, namely, Scutellaria Radix, Coptidis Rhizoma, and Glycyrrhizae radix et rhizoma. It could be used to treat the coke fire, big swollen head, swollen throat, ear nose mouth heart heat, and boil the virus, owing to the functions of

\section{Springer Open}


clearing away heat and toxic material (Wu 1957). Semibionic extraction (SBE) method is an innovative extraction technology for compound prescriptions of TCM administered through the digestive tract, which combines the overall and molecular drug research together. SBE simulates the process of oral administration and drug transport through the gastrointestinal tract and embodies the characteristics of the comprehensive components of TCM (Wang et al. 2016b, Xu and Shi 2011). Based on SBE, semi-bionic enzyme extraction (SBEE) method is a further "biomimetic" research design using bio-enzymes to extract pharmacodynamic substances of TCM, especially compound prescriptions. Bio-complex enzymes (cellulase, pectinase, and some proteases) and digestive enzymes can destroy cell walls of herbs, which could reduce the resistance of mass transfer barriers for the diffusion of active ingredients from intracellular to extracting media, thus facilitating the dissolution of active ingredients. As the extraction temperature decreases, the extraction conditions close to human gastrointestinal environment and the destruction of active ingredients at high temperature can be avoided.

At present, researches on SBE methods mainly focuses on the optimization of the SBE process (Wang et al. 2011; Zhang et al. 2013), comparison of different extraction methods (Wang et al. 2013a), comparison of pharmacodynamics (Wang et al. 2016b), ownership study of HPLC fingerprints (Wang et al. 2016c), et al. On these bases, the effect of SBEE method was further studied. Previous studies have shown that cellulase treatment could significantly improve the extraction rate of bioactive compounds in TCM, comparing with the traditional water or alcohol extraction process (Dai et al. 2014; Wang et al. 2007). It was also found in our previous research that SBEE method had significant advantages. Compared with SBE method, the extraction rate of berberine and glycyrrhetinic acid in Erhuang decoction could be improved by SBEE method. Besides, the bioavailability of baicalin, berberine, palmatine, and glycyrrhetinic acid in Erhuang decoction extracted by SBEE method was higher. What's more, the serum fingerprints showed that the characteristic peaks of SBEE extract were more than that of SBE extract at the same dosage (Wang 2014).

Current research methods for the bioactive compounds or chemical components in TCMs are various, such as pharmacological screening (Gao et al. 2019; Li et al. 2019), cell culture-based assays, equilibrium dialysis, microdialysis, ultrafiltration, and chromatographic methods (Fu et al. 2019; Huang et al. 2004). Among them, chromatographic methods are usually applied with other screening methods, for example, ultra-high-performance liquid chromatography (UPLC) coupled with quadrupole-timeof-flight mass spectrometry (Q-TOF-MS) analysis (Yuan et al. 2016), equilibrium dialysis/HPLC (Xiao et al. 2014), microdialysis/HPLC-MS/MS or HPLC-Q-TOF-MS/MS (Su et al. 2005; Wang et al. 2016a; Wang et al. 2013b), and GC-MS techniques (Zhang et al. 2007a).

In this research, Erhuang decoction was extracted by SBEE method and studied for the attribution and identification of absorbed components in rat serum after oral administration. A HPLC-DAD-ESI-MS method was used to establish the fingerprints and analyze the bioactive compounds of Erhuang decoction. The results may provide an evidence for the clinical feasibility and advantage of Erhuang decoction.

\section{Materials and methods}

\section{Chemicals and reagents}

Scutellariae radix, Coptidis rhizoma, and Glycyrrhizae radix et rhizoma were purchased from Jianlian Traditional Chinese Drug Store (Jinan, China) and authenticated by Prof. Zhang of College of Pharmacy, Shandong University of Traditional Chinese Medicine. Enzyme I, straw decomposing agent, was achieved from Inner Mongolia Shantang Agricultural Science and Technology Co., Ltd. (Ulanhot, China). Enzyme II, cellulase, was obtained from Suhequan Biology Co., Ltd. (Weifang, China). Enzyme III (pepsin) and enzyme IV (pancreatin) were bought from Hubei Julongtang Biotechnology Development Co., Ltd. (Wuhan, China). Purity of all the reference substance was over $98 \%$. Baicalin was purchased from Shandong Institutes for Food and Drug Control (Jinan, China). Baicalein, berberine hydrochloride, glycyrrhizic acid, glycyrrhetinic acid, and liquiritin were bought from National Institutes for Food and Drug Control (Beijing, China). Palmatine hydrochloride and liquiritigenin were obtained from Nanchang Beta Biotechnology Co. Ltd. (Nanchang, China). Methanol and acetonitrile (Merck, Germany) were of HPLC grade, and deionized water was purified by a Milli-Q system (Millipore, Bedford, MA, USA). Ammonium acetate, acetic acid, and other chemicals and reagents used in this study were of analytical grade.

Animals: male Sprague-Dawley rats, weighting $200 \pm$ $20 \mathrm{~g}$, were provided by the Experimental Animal Center of Shandong University of Traditional Chinese Medicine (Jinan, China). The animals were fed with food and water ad libitum in a breeding room at $25 \pm 2{ }^{\circ} \mathrm{C}$ with humidity of $60 \pm 5 \%$. They were housed for at least 1 week acclimation and were fasted overnight prior to the experiments. The animal care and all procedures were in accordance with the National Institutes of Health Guide for Care and Use of Laboratory Animals.

\section{Preparation of Erhuang decoction extracts by SBEE method}

The Erhuang decoction extracts were prepared by SBEE methods (Wang et al. 2013a). Briefly, appropriate amount 
of Scutellariae radix, Coptidis rhizoma, and Radix liquiritiae with the ratio of 1:1:1 was powdered and pre-treated by $1.0 \%$ enzyme I for $36 \mathrm{~h}$ and $1.0 \%$ enzyme II for $36 \mathrm{~h}$. Then Scutellariae radix and Radix liquiritiae were decocted together, while Coptidis rhizoma was decocted separately. The extraction procedure of the first step was $0.5 \%$ enzyme III, $\mathrm{pH} 2.0$, and extraction time was $2.0 \mathrm{~h}$. The second process was $\mathrm{pH} 6.5$ and extraction time was $1.5 \mathrm{~h}$. The third was $1.0 \%$ enzyme IV, $\mathrm{pH} 9.0$, and extraction time was $1.5 \mathrm{~h}$. At last, the extracting solution was filtered by hollow fiber membrane, centrifuged for $25 \mathrm{~min}$ at $3500 \mathrm{r} \cdot \mathrm{min}^{-1}$, concentrated and mixed to obtain $1.0 \mathrm{~g} \cdot \mathrm{mL}^{-1}$ Erhuang decoction extracts. As a contrast, the single decoction extracts from Scutellaria baicalensis, Coptis chinensis, or licorice were prepared in the same methods.

\section{Preparation of reference solutions}

Various accurate amounts of reference compounds (5.47 mg baicalin, $1.98 \mathrm{mg}$ baicalein, $2.60 \mathrm{mg}$ liquiritin, $0.28 \mathrm{mg}$ liquiritigenin, $2.64 \mathrm{mg}$ palmatine hydrochloride, $2.56 \mathrm{mg}$ berberine hydrochloride, $5.02 \mathrm{mg}$ glycyrrhizic acid monoammonium salt, $1.99 \mathrm{mg}$ glycyrrhetinic acid) were respectively dissolved into $10 \mathrm{~mL}$ methanol to prepare the primary stock solutions. Appropriate volume of these stock solutions was evaporated under a light stream of nitrogen, and the residues were redissolved in $10 \mathrm{~mL}$ methanol to obtain the mixed reference solution containing eight components. The final concentrations were $0.547 \mathrm{mg} \cdot \mathrm{mL}^{-1}$ baicalin, $0.297 \mathrm{mg} \cdot \mathrm{mL}^{-1}$ baicalein, $0.078 \mathrm{mg} \cdot \mathrm{mL}^{-1}$ liquiritin, $0.056 \mathrm{mg} \cdot \mathrm{mL}^{-1}$ liquiritigenin, $0.132 \mathrm{mg} \cdot \mathrm{mL}^{-1}$ palmatine hydrochloride, $0.384 \mathrm{mg} \cdot \mathrm{mL}^{-1}$ berberine hydrochloride, $0.251 \mathrm{mg} \cdot \mathrm{mL}^{-1}$ glycyrrhizic acid monoammonium salt, and $0.0597 \mathrm{mg} \cdot \mathrm{mL}^{-1}$ glycyrrhetinic acid, respectively.

\section{Animal treatments and preparation of rat serum samples} Thirty male SD rats were divided into five groups and orally administrated: group I, Erhuang decoction concentrated extract $\left(15 \mathrm{~g} \cdot \mathrm{kg}^{-1}\right.$ ) (Wang et al. 2017); group II, Scutellaria baicalensis extract $\left(5 \mathrm{~g} \cdot \mathrm{kg}^{-1}\right)$; group III, Coptis chinensis extract $\left(5 \mathrm{~g} \cdot \mathrm{kg}^{-1}\right)$; group IV, licorice extract $\left(5 \mathrm{~g} \cdot \mathrm{kg}^{-1}\right)$; group $\mathrm{V}$, equal volume of $0.9 \% \mathrm{NaCl}$ (normal saline). The blood was collected from the orbital venous plexus into $1.5 \mathrm{~mL}$ polythene tubes after treatment for $0.5 \mathrm{~h}$, and was kept still for $1 \mathrm{~h}$ to isolate $200 \mu \mathrm{L}$ serum.

Methanol, equivalent to five times the volume of plasma, and $20 \mu \mathrm{L}$ ammonium acetate solution (0.02 $\mathrm{mmol} \cdot \mathrm{L}^{-1}, \mathrm{pH} 4.0$ ) were added to each obtained serum to precipitate protein by vortex for $5 \mathrm{~min}$. Then, the samples were centrifuged at $10000 \mathrm{rpm}$ for $20 \mathrm{~min}$, and the supernatant was evaporated at $40{ }^{\circ} \mathrm{C}$ under a gentle stream of nitrogen using a EVA50A Nitrogen Evaporators (Polytech Instrument Ltd., China). The residues were redissolved in $100 \mu \mathrm{L}$ methanol and filtered through a $0.22-\mu \mathrm{m}$ filter for next analysis.

\section{Chromatographic and mass spectrometry conditions}

The fingerprints of extracts from Erhuang decoction, single Scutellaria baicalensis, Coptis chinensis, or licorice, as well as the drug containing serum after administration of these extracts, were prepared using a HPLC-DAD-ESI-MS approach. And the speculation and identification of relevant compounds contained in the serum were conducted.

The analyses were carried out using an Agilent 1100 liquid chromatography system, equipped with an Agilent 1100 Trap VL MS system (Agilent Technologies, USA) and a DAD detector (G1315D, Agilent Technologies, USA). The separation was performed on an Diamonsil C18 column $(250 \mathrm{~mm} \times 4.6 \mathrm{~mm}, 5 \mu \mathrm{m})$ at $25{ }^{\circ} \mathrm{C}$, with mobile phase consisting of solvent $\mathrm{B}$ (acetonitrile) and solvent A (0.02 mmol. $\mathrm{L}^{-1}$ ammonium acetate, $\left.\mathrm{pH} 4.0\right)$. Gradient elution was as follows: initial 0-30 $\mathrm{min}, 10$ 25\% (B); 30-42 min, 25-30\% (B); 42-55 min, 30-30\% (B); $55-70 \mathrm{~min}, 30-50 \%$ (B); 70-90 $\mathrm{min}, 50-100 \%$ (B), 90-100 min, $100-100 \%$ (B). The flow rate of the mobile phase was $0.5 \mathrm{~mL} \cdot \mathrm{min}^{-1}$. The injection volume was $20 \mu \mathrm{L}$, and the wavelength of the UV-detection was determined at $265 \mathrm{~nm}$.

The mass spectrometric detection was operated with ESI source in positive and negative ionization switching mode. The positive and negative ion modes were run separately. Tune parameters were as follows: ion spray voltage $-4.5 \mathrm{kV}\left(\mathrm{ESI}^{-}\right) / 4.5 \mathrm{kV}\left(\mathrm{ESI}^{+}\right)$; nebulizer gas pressure $30 \mathrm{psi}$; desolvation gas flow $10.00 \mathrm{~L} \cdot \mathrm{min}^{-1}$; ion source temperature $350{ }^{\circ} \mathrm{C}$; source fragmentation voltage $150 \mathrm{~V}$. The analyzer scanned over the mass range $\mathrm{m} / \mathrm{z}$ $100-1500$.

\section{Results and discussion}

The attribution of characteristic peaks in drug containing serum fingerprints of Erhuang decoction extract

Figure 1 displays the chromatograms of mixed reference compounds. Figure 2 shows the chromatograms of serum containing the extracts of Erhuang decoction, single Scutellaria baicalensis, Coptis chinensis, or licorice. To make a comparison, the chromatograms in vitro were exhibited together.

When a characteristic peak in the fingerprint of drugcontaining serum corresponds to a peak in a single drug, it may attribute to an original form compound existing in the single drug. If a characteristic peak exists in the fingerprints of the serum containing a single drug but does not exist in the fingerprints of the single drug, it may come from the metabolites of the single drug in vivo. When a characteristic peak exists in fingerprints of serum containing the whole prescription but does not 


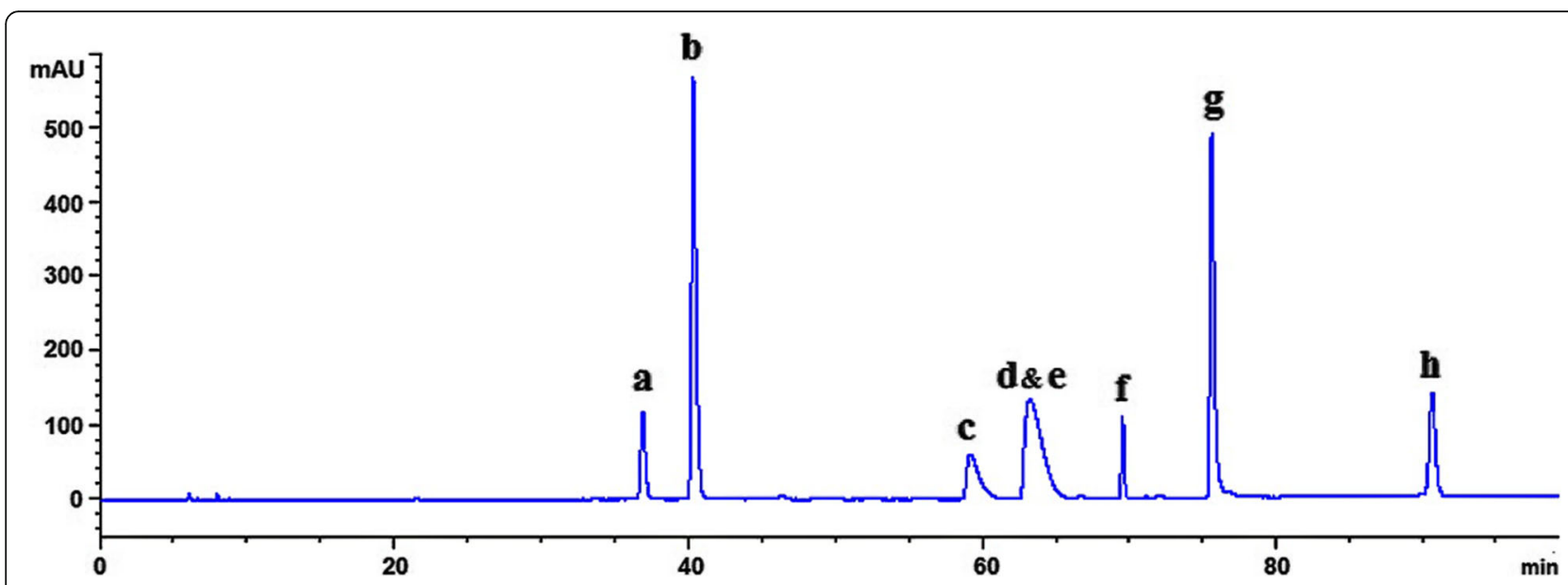

Fig. 1 The chromatograms of mixed reference solution: (a) liquiritin, (b) baicalin, (c) palmatine hydrochloride, (d) berberine hydrochloride, (e) liquiritigenin, (f) glycyrrhizic acid, (g) baicalein, (h) glycyrrhetinic acid

exist in fingerprints of serum containing any single drug, it may originate from a new substance produced by the metabolism of the whole prescription in vivo.

By comparing the fingerprint of serum containing Erhuang decoction extract with the fingerprint in vitro (Fig. $2 \mathrm{~b}$ and $\mathrm{c}$ ), 24 peaks that migrate into the blood from Erhuang decoction were detected. It was inferred that 20 peaks (peaks $1,2,4,5,7,9,10,11,13,14,15,16$, $17,18,19,20,21,22,23$, and 24) were directly absorbed into the blood as original form compounds of the prescription. In comparison with the chromatograms of mixed reference solution (Fig. 1), peaks 10, 11, 18, 19, 20 , and 24 were identified as liquiritin, baicalin, palmatine, berberine (and liquiritigenin), baicalein, and glycyrrhetinic acid, respectively. At the retention time of glycyrrhizic acid, no corresponding peak existed in the fingerprint of serum containing licorice (Fig. 2i) or Erhuang decoction (Fig. 2c), so it could be inferred that glycyrrhizic acid was decomposed into glycyrrhetinic acid when being absorbed into the blood.

From the fingerprints of drug-containing serum after administration of Erhuang decoction extracts, some characteristic peaks originated from the prototype components of single or multiple drugs in Erhuang decoction. Peaks 13, 20, and 23 came from the original form compounds existing in Scutellaria baicalensis. Peak 15, 18 originated from Coptis chinensis, and peaks 1, 2, 10,21 , and 24 were the prototype components of licorice. Peaks 11 and 22 came from Scutellaria baicalensis and licorice, and peak 19 originated from Coptis chinensis and licorice. Peaks 16 and 17 came from Scutellaria baicalensis, Coptis chinensis, and licorice. However, some characteristic peaks were the metabolites of Erhuang decoction that migrated into the blood or new compound produced by the metabolism process. Peak 8 was the metabolites of both Scutellaria baicalensis and licorice. What's more, as shown in Table 1 , some characteristic peaks not only came from original form compounds, but also the metabolites of Scutellaria baicalensis, Coptis chinensis, or licorice (peaks 3, 4, 5, 7, 9, 14, and 24). According to the MS/MS data and serum fingerprints of Erhuang decoction, peaks 6 and 12 displayed some changes in peak area and were consistent with those of mass spectrometry in blank serum, so it could be inferred that they were endogenous substances in the serum.

Identification of the components in serum samples after oral administration of Erhuang decoction extracts in rats By analyzing the MS data and comparing with the mixed reference solution, the components in serum samples after oral administration of Erhuang decoction extracts were further studied on their identification. The TIC chromatogram of drug-containing serum after administration of the Erhuang decoction extracts is shown in Fig. 3.

\section{The identification of migratory components into the blood relating to mixed reference compounds} Utilizing the technique of HPLC-DAD-MS, characteristic peaks in the fingerprints of serum containing Erhuang decoction extract (peaks 10, 11, 18, 19, 20, and 24 in Fig. 2c) were identified by corresponding to the retention time and mass spectra of the reference compounds.

Table 2 displays the MS/MS data of (+) ESI-MS spectra and (-) ESI-MS spectra, and the identification results of the constituents of Erhuang decoction. The MS/MS data shows that peak 10 lost a fragment ion of glucose (162) and peak 11 lost a fragment ion of glucuronic acid (176, glc A) in the cracking process. Peak 20 split into two fragment ions according to the RDA (Retro Diels-Alder) 


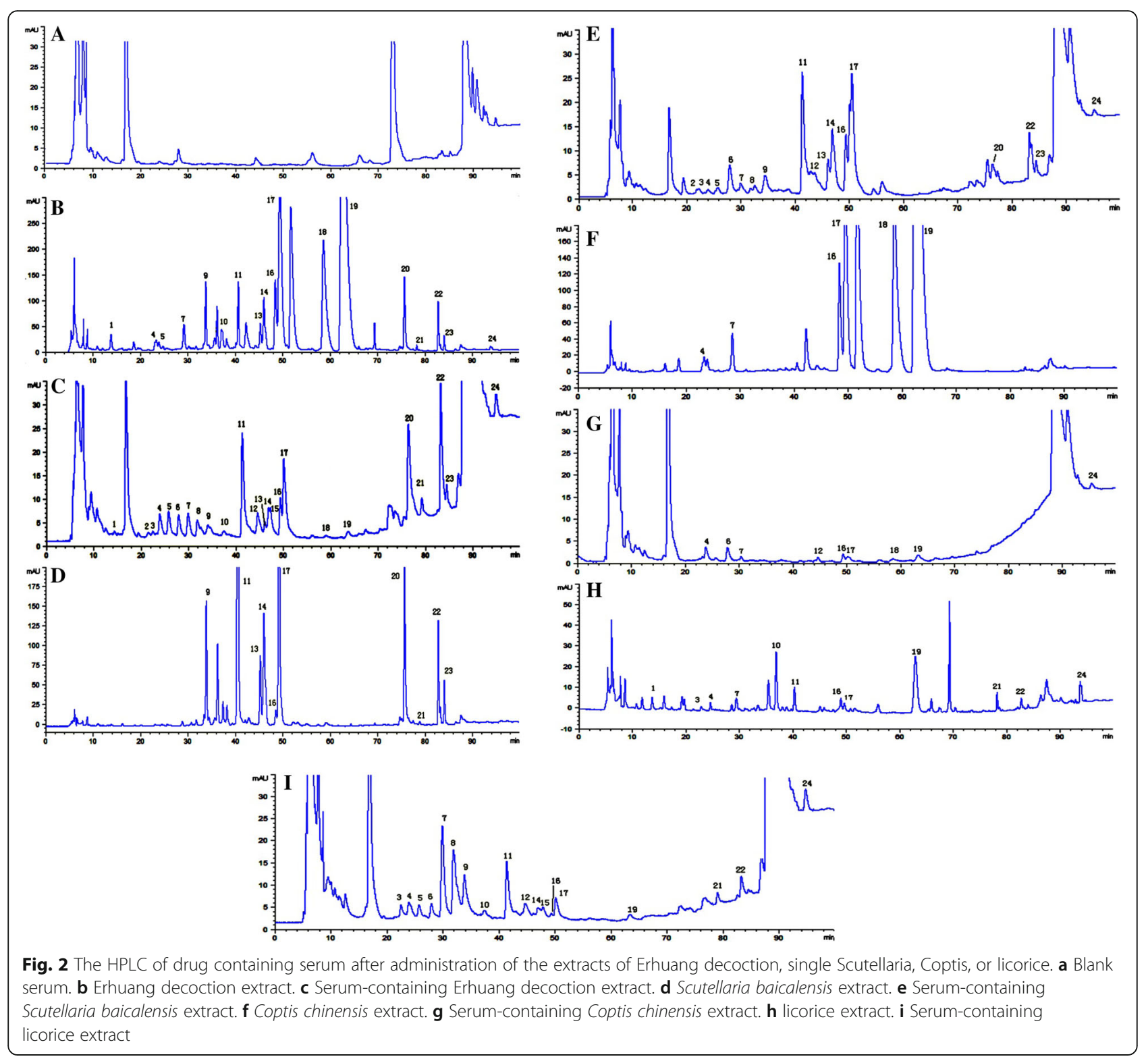

reaction in cracking process (Fig. 4). According to the MS data, the molecular weights of the constituents corresponding to peaks $10,11,18,20$, and 24 were confirmed to be $418,446,352,270$, and 470, respectively. Peaks 10 (liquiritin, $\mathrm{C}_{21} \mathrm{H}_{22} \mathrm{O}_{9}$ ), 11 (baicalin, $\mathrm{C}_{21} \mathrm{H}_{22} \mathrm{NO}_{4}$ ), 18 (palmatine, $\mathrm{C}_{21} \mathrm{H}_{22} \mathrm{NO}_{4}$ ), 20 (baicalein, $\mathrm{C}_{15} \mathrm{H}_{10} \mathrm{O}_{5}$ ), and 24 (glycyrrhetinic acid, $\mathrm{C}_{30} \mathrm{H}_{46} \mathrm{O}_{4}$ ) were identified by comparing the retention time and MS data with those of the reference compounds (a, b, c, g, and h), respectively.

The MS/MS data of peak 19: in positive ion ESI mode, two parent ions, 336.1 $[\mathrm{M}]^{+}$and $257.0[\mathrm{M}+\mathrm{H}]^{+}$and their corresponding fragment ions, $321.1\left[\mathrm{M}-\mathrm{CH}_{3}\right]^{+}$,

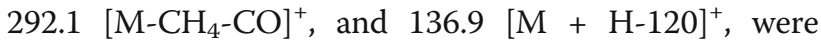
detected; in negative ion ESI mode, the parent ion was $254.8[\mathrm{M}-\mathrm{H}]^{-}$, and the fragment ion was $134.6[\mathrm{M}-\mathrm{H}-$
$120]^{-}$. It could be deduced that the molecular weights of the two components were 336 and 256, respectively. And by comparing with the reference compounds $\mathrm{d}$ and e, peak 19 was referred to be composed of berberine $\left(\mathrm{C}_{20} \mathrm{H}_{18} \mathrm{NO}_{4}\right)$ and liquiritigenin $\left(\mathrm{C}_{15} \mathrm{H}_{12} \mathrm{O}_{4}\right)$. Liquiritigenin split into two fragment ions according to the RDA reaction. Under the condition of liquid chromatography in this study, berberine and liquiritigenin could not be completely separated due to the polarity and $\mathrm{pH}$ value of the mobile phase. The two constituents existed as the same chromatographic peak (peak 19). Under the condition of mass spectrometry, peak 19 was given in positive ion ESI mode and had two molecular ion peaks, 336.1 and 257.0, which were consistent with the fragment ions of the reference compounds. Furthermore, by analyzing 
Table 1 Spectra data and the attribution results of 24 peaks of serum fingerprints after oral administration of Erhuang decoction

\begin{tabular}{|c|c|c|c|}
\hline No. & $t_{\mathrm{R}} / \min$ & $\lambda_{\max } / \mathrm{nm}$ & Attribution \\
\hline 1 & 14.3 & 280 & Original form compounds existed in licorice \\
\hline 2 & 21.4 & 248 & Original form compounds existed in licorice \\
\hline 3 & 22.4 & - & Original form compounds existed in licorice and the metabolites of Scutellaria baicalensis \\
\hline 4 & 23.8 & 252 & Original form compounds existed in Coptis chinensis and licorice; metabolites of Scutellaria baicalensis \\
\hline 5 & 25.7 & 260 & Original form compounds existed in licorice and the metabolites of Scutellaria baicalensis and licorice \\
\hline 6 & 27.8 & 275 & Control serum \\
\hline 7 & 29.8 & 275 & Original form compounds existed in Coptis chinensis and licorice; metabolites of Scutellaria baicalensis \\
\hline 8 & 31.7 & 272 & Metabolites of licorice and Scutellaria baicalensis \\
\hline 9 & 34.5 & 290 & Original form compounds existed in Scutellaria baicalensis and metabolites of licorice \\
\hline 10 & 37.7 & 276 & Original form compounds existed in licorice \\
\hline 11 & 41.3 & 277 & Original form compounds existed in Scutellaria baicalensis and licorice \\
\hline 12 & 44.5 & 360 & Control serum \\
\hline 13 & 46.0 & 270 & Original form compounds existed in Scutellaria baicalensis \\
\hline 14 & 46.8 & 275 & Original form compounds existed in Scutellaria baicalensis and metabolites of licorice \\
\hline 15 & 47.2 & 270 & Original form compounds existed in Coptis chinensis \\
\hline 16 & 49.3 & 276 & Original form compounds existed in Scutellaria baicalensis, Coptis chinensis, and licorice \\
\hline 17 & 50.0 & 275 & Original form compounds existed in Scutellaria baicalensis, Coptis chinensis, and licorice \\
\hline 18 & 59.3 & 273 & Original form compounds existed in Coptis chinensis \\
\hline 19 & 63.6 & 274 & Original form compounds existed in Coptis chinensis and licorice \\
\hline 20 & 76.3 & 277 & Original form compounds existed in Scutellaria baicalensis \\
\hline 21 & 79.0 & 265 & Original form compounds existed in licorice \\
\hline 22 & 83.1 & 273 & Original form compounds existed in Scutellaria baicalensis and licorice \\
\hline 23 & 84.4 & 272 & Original form compounds existed in Scutellaria baicalensis \\
\hline 24 & 94.4 & 250 & Original form compounds existed in Licorice \\
\hline
\end{tabular}

Note: $\lambda_{\max }$ means maximum absorption wavelength

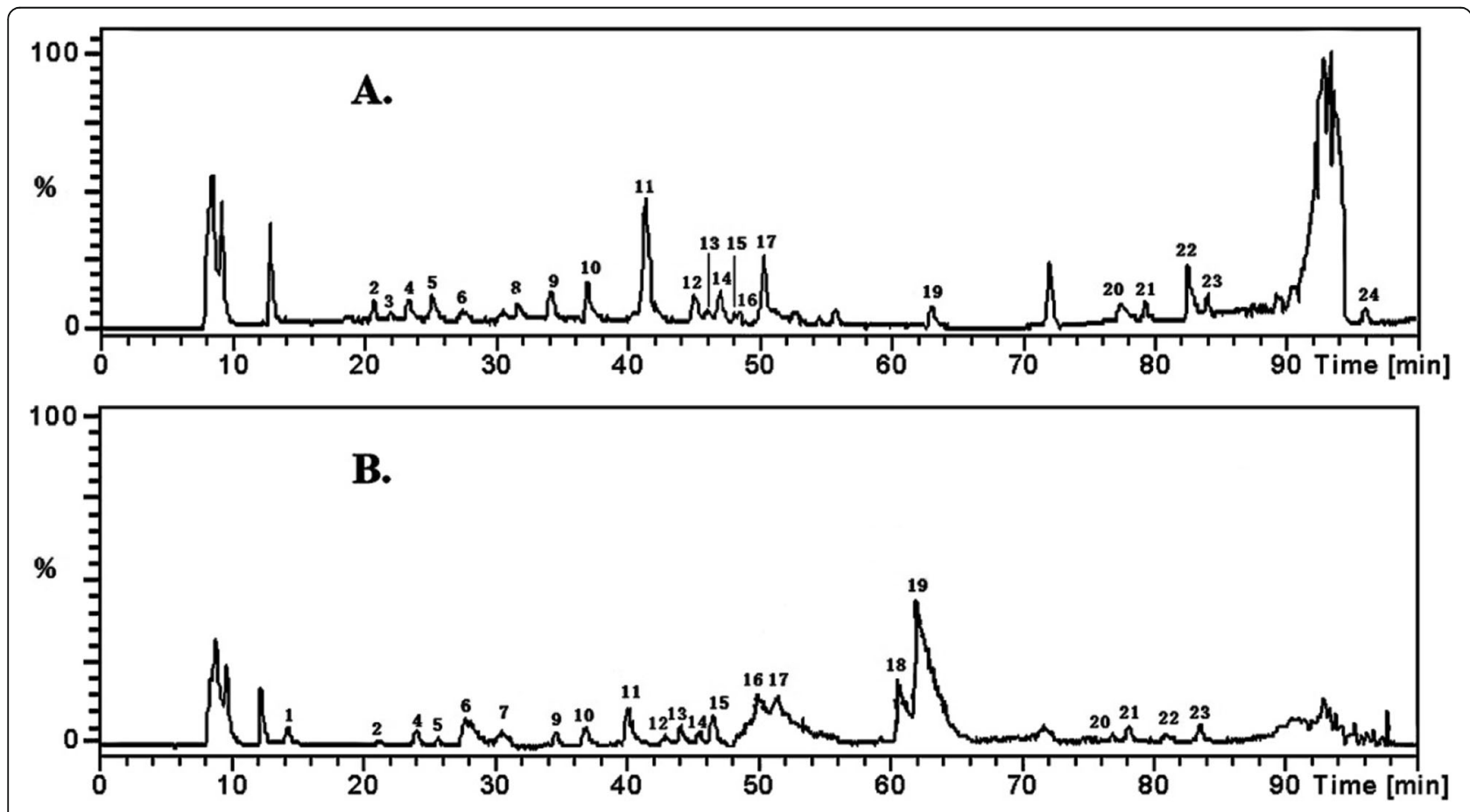

Fig. 3 The TIC of drug-containing serum after administration of Erhuang decoction extracts. a TIC in negative ESI mode. b TIC in positive ESI mode 
Table 2 MS/MS data of (+) ESI-MS spectra and (-) ESI-MS spectra, and the identification results of the constituents of Erhuang decoction

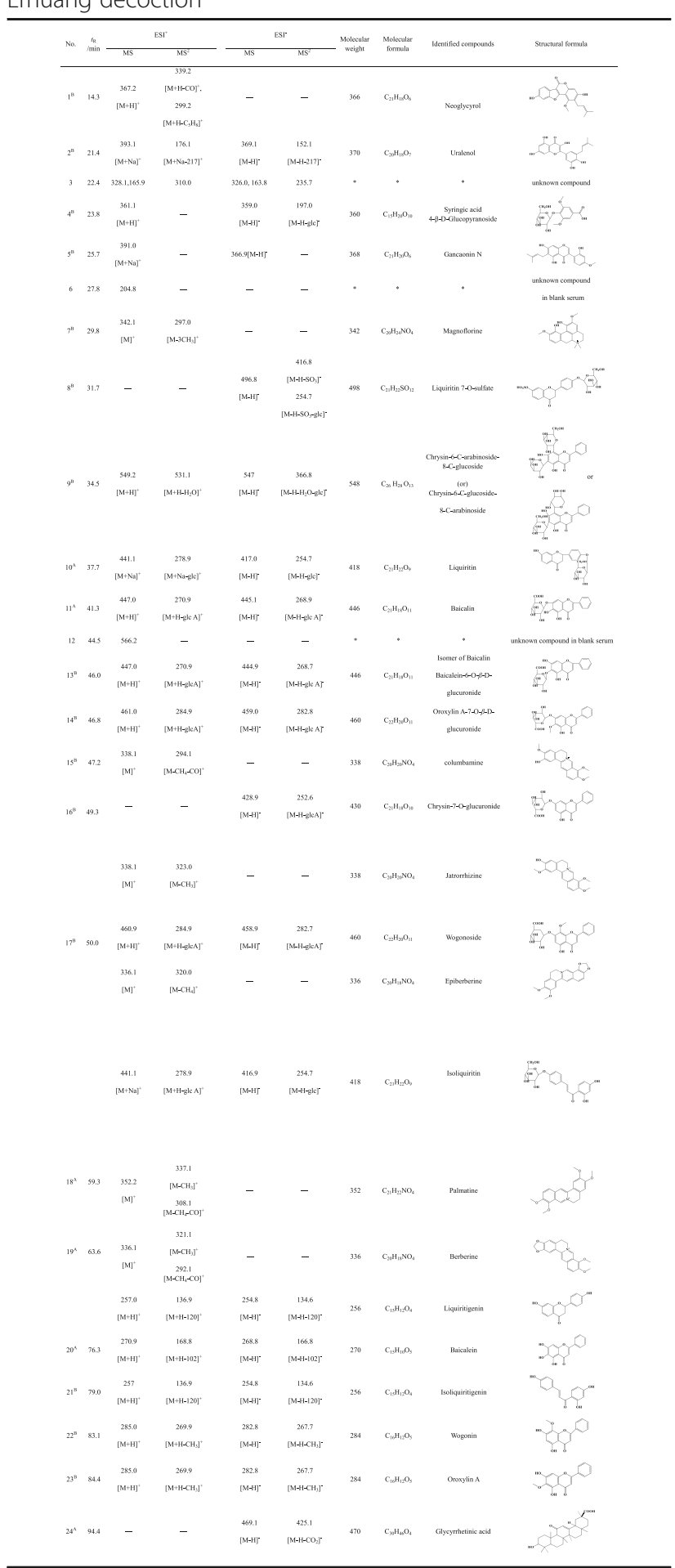

Note: "—" means no effective spectral and mass spectrometric data were obtained

glc glucose, glc $A$ glucuronic acid

*Means not identified

${ }^{A}$ Means the components determined by comparing with the reference compounds

${ }^{\mathrm{B}}$ Means the components determined by retrieving the reference literatures. the MS/MS data, peak 19 was definitely attributed to berberine and liquiritigenin.

The reference compound $f$ is glycyrrhizic acid $\left(\mathrm{C}_{42} \mathrm{H}_{62} \mathrm{O}_{16}\right)$ with a molecular weight of 822 and a retention time of $69.3 \mathrm{~min}$ (Fig. 1). The MS/MS spectrum shows $821.6[\mathrm{M}-\mathrm{H}]^{-}, 803.3\left[\mathrm{M}-\mathrm{H}-\mathrm{H}_{2} \mathrm{O}\right]^{-}$, and $645.3[\mathrm{M}-$ $\mathrm{H}$-glc A] $]^{-}$. As shown in Fig. $2 \mathrm{~b}$ and c, a similar peak (peak a11) appeared at the corresponding position in the fingerprint of Erhuang decoction extracts in vitro, while no corresponding chromatographic peaks were found in the fingerprint in vivo. In addition, this peak was only attributed from glycyrrhiza uralensis and was determined to be glycyrrhizic acid.

In this study, glycyrrhizic acid could not be detected in liquid chromatography and mass spectrometry after being absorbed into the blood. As the dose of glycyrrhizic acid was small and the composition of the compound prescription was complex, the absorption and metabolism of glycyrrhizic acid was possibly interfered. It has been reported that only when glycyrrhizic acid is orally administration at more than $50 \mathrm{mg} \cdot \mathrm{kg}^{-1}$, can it be detected in blood owing to its low oral bioavailability. By the hydrolysis of intestinal flora, glycyrrhizic acid is converted into glycyrrhetinic acid (peak 24) which is an aglycone of glycyrrhizic acid that can be easily detected in vivo (Cantelli-Forti et al. 1994; Yamamura et al. 1995).

\section{The identification of other components migrating into the blood}

Combined with the MS/MS data, molecular weight, and corresponding data recorded in literatures, peaks 1, 2, 4, 5, 7 , and 8 were identified as neoglycyrol, uralenol, syringic acid 4- $\beta$-D-glucopyranosidel, gancaonin $\mathrm{N}$, magnoflorine, and liquiritin 7-O-sulfate, respectively (Dong et al. 2014; Fang et al. 2016; Li 2013; Zhao et al. 2016). After being absorbed into the blood, 7-OH of liquiritin was sulfated to form the metabolite liquiritin 7-O-sulfate (peak 8), which lost a fragment ion of $\mathrm{SO}_{3}(80)$ and glucose (162) in the cracking process (Fig. 5). Besides, peak 9 was identified as chrysin-6-C-Arabia sugar-8-C-glucoside or chrysin glycoside-6-C-glucose-8-C-Arabia (Liu et al. 2012).

The MS/MS data of peak 13 and its molecular weight were consistent with the MS/MS data of peak 11 (baicalin). These results also indicated that the mother nucleus of this isomer is identical to the aglycone of baicalin, and the isomerization may occur in the glycosyl group. What's more, it has been reported that isomers of baicalin exist in Scutellaria baicalensis (Liu et al. 2012), so peak 13 might be identified as the isomer of baicalin (Baicalein-6-O- $\beta$-D-glucuronide).

The MS/MS data of peak 14 was 461.0 $[\mathrm{M}+\mathrm{H}]^{+}$, $284.9[\mathrm{M}+\mathrm{H} \text {-glc A }]^{+}, 459.0[\mathrm{M}-\mathrm{H}]^{-}, 282.8[\mathrm{M}-\mathrm{H}$-glc $\mathrm{A}]^{-}$, and the fragment ion is glucosiduronic acid. The 


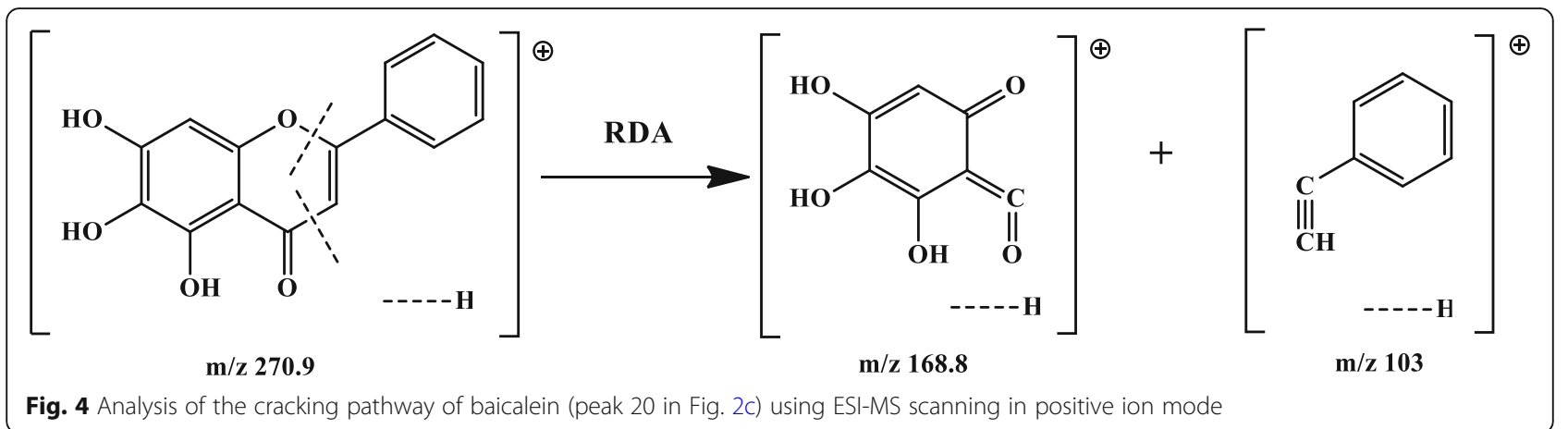

MS/MS data of peak 17 was $460.9[\mathrm{M}+\mathrm{H}]^{+}, 284.9[\mathrm{M}+$ $\mathrm{H}$-glc $\mathrm{A}]^{+}$. Therefore, the molecular weight of the two components (peaks 14 and 17) were confirmed to be 460 and 459.9, respectively. It has been reported that there is a pair of isomers weighting 460 in Scutellaria (Han et al. 2007), oroxylin A-7-O- $\beta$-D-glucuronide, and wogonoside. Under the chromatographic conditions of this study, the polarity of wogonoside was smaller than oroxylin A-7-O- $\beta$-D-glucuronide, which illustrated that the retention time of wogonoside was longer than oroxylin A-7-O- $\beta$-D-glucuronide. Results showed that the retention times of peaks 14 and 17 were 46.8 and 49.2 min, respectively. Then, combined with the relevant literature on mass-to-charge ratio, wavelength, and retention time (Zhang et al. 2007b), peaks 14 and 17 were respectively identified as oroxylin A-7-O- $\beta$-D-glucuronide and wogonoside, respectively. In addition, comparing with the mass-to-charge ratio in literature, $336.1[\mathrm{M}]^{+}$of peak 17 may be the molecular ion peak of epiberberine (Chen et al. 2008). As the MS/MS data of peak $17\left(416.9[\mathrm{M}-\mathrm{H}]^{-}, 254.7\left[^{\left.\mathrm{M}-\mathrm{H}-\mathrm{glc}]^{-}\right) \text {was partially }}\right.\right.$ similar to the MS/MS data of peak $10\left(417.2[\mathrm{M}-\mathrm{H}]^{-}\right.$, $\left.254.9[\mathrm{M}-\mathrm{H} \text {-glc }]^{-}\right)$, peak 17 was inferred to be isoliquiritin (Zhao et al. 2016). Therefore, peak 17 was finally attributed to three components, wogonoside, epiberberine, and isoliquiritin.

The MS/MS data of peak 15 was $338.1[\mathrm{M}]^{+}, 294.1$ $\left[\mathrm{M}-\mathrm{CH}_{4}-\mathrm{CO}\right]^{-}$, and its molecular weight was 338 (Li 2013). And by corresponding data recorded in literature, this peak was identified as columbamine (Li 2013). Peak 16 was deduced to originate from jatrorrhizine in accordance with its MS/MS data $\left(338.1[\mathrm{M}]^{+}, 323.0\right.$ [M$\left.\mathrm{CH}_{3}\right]^{+}$) (Zuo et al. 2014). And the fragment ions, 252.6 [M-H-glc A $]^{-}$, came from $428.9[\mathrm{M}-\mathrm{H}]^{-}$by losing the fragment of glucuronic acid, which pointed out that its molecular weight was 430 . So peak 16 was identified as chrysin-7-O-glc A, comparing with the literature (Liu et al. 2012). The MS/MS data of peak $21\left(254.8[\mathrm{M}-\mathrm{H}]^{-}\right.$, $\left.134.6[\mathrm{M}-\mathrm{H}-120]^{-}\right)$was consistent with that of liquiritigenin $\left(254.8[\mathrm{M}-\mathrm{H}]^{-}, \quad 134.6[\mathrm{M}-\mathrm{H}-120]^{-}\right)$, which indicated that peak 21 was attributed to liquiritigenin. What's more, it has been reported that a pair of isomers, liquiritigenin and isoliquiritigenin, existed in licorice, and their mass spectrometry data were consistent (Zhao et al. 2016). The molecular weight of peaks 22 and 23 was inferred as 284, according to their MS data in Table 2. It has been reported that a pair of isomers, wogonin and oroxylin A, with molecular weight of 284 , existed in Scutellaria baicalensis (Liu et al. 2012). They are the aglycone of wogonoside and oroxylin A-7-O- $\beta$-D-glucuronide, respectively. In comparison with the literature, peaks 22 and 23 were speculated as wogonin and oroxylin A, respectively. Although the MS/MS spectra of peaks 3,6 , and 12 were also analyzed, the compounds could not be identified for lacking relevant references or mass spectrometry data. Thus, these three peaks were only attributed according to the chromatographic and spectral data of the fingerprints of the single herb or drug-containing serum (Table 1).

In general, the attribution of characteristic peaks should be determined by combining the chromatographic, spectral, and mass spectrometric data

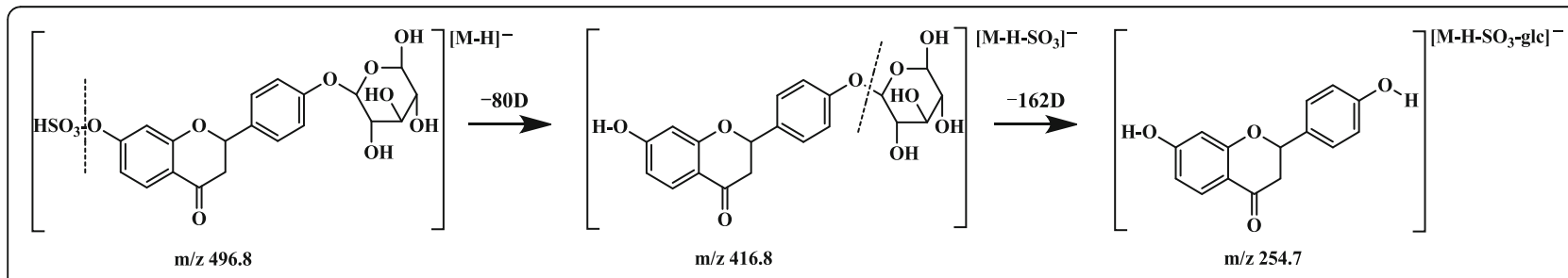

Fig. 5 Analysis of the cracking pathway of liquiritin 7-O-sulfate (peak 8 in Fig. 2c) using ESI-MS scanning in negative ion mode 
comprehensively. Peaks 4, 5, 7, 8, 9, 11, 14, 15, 16, and 22 were identified or inferred partially by analyzing the mass spectrometry and comparing with reference compounds and literatures. However, the results were not entirely consistent with the chromatographic attribution. For example, peak 11 was determined to be baicalin by MS analysis, but it was attributed as the original form compound existed in Scutellaria baicalensis and licorice according to the chromatography analysis. The differences might be due to the absorption inhibition of original form compounds in the compound prescription, which resulted in low serum content, undetectable, or unsatisfying ionization effect of the components in this mass spectrometry condition. Therefore, the attribution of the peak cannot simply base on the basis of MS/MS data.

\section{Conclusions}

In this study, the characteristic peaks of serum fingerprint after oral administration of Erhuang decoction extracted by SBEE method were attributed and identified by HPLC-DAD-ESI-MS. Firstly, the characteristic peaks of drug-containing serum fingerprint were compared with the in vitro and in vivo fingerprints of each single drug in the prescription, to attribute the constituents migrating into the blood, including the original form compounds, metabolic components, or new components produced by metabolism of each characteristic peak were determined. Then, in comparison with the standard references, seven components in Erhuang decoctioncontaining serum were identified, and eighteen were speculated comparing with literatures. Besides, molecular weights of some bioactive constituents were obtained, and the model of researching on the effective components of Erhuang decoction was improved. Therefore, the foundation of developing a new modern formulation and studying the action mechanism of the prescription has been laid.

\section{Abbreviations \\ TCM: Traditional Chinese medicine; YCHT: Yin Chen Hao Tang; SBE: Semi- bionic extraction; SBEE: Semi-bionic enzyme extraction; UPLC: Ultra-high- performance liquid chromatography; Q-TOF-MS: Quadrupole-time-of-flight mass spectrometry}

\section{Acknowledgements}

Not applicable.

\section{Authors' contributions}

This research work was planned by YW, XS, and ZZ. The experiments and analysis were executed by JW, DZ, NX, and CZ. JW and DZ were the major contributor in writing the manuscript. All authors read and approved the final manuscript.

\section{Funding}

This work was supported by the Shandong Provincial Natural Science Foundation (No. ZR2018LH020), Scientific Research Foundation of the Higher Education Institutions of Shandong Province (No. J18K035), and Youth Innovation Team of Scientific Research Foundation of the Higher Education Institutions of Shandong Province, China (No. 2019KJM006).

\section{Availability of data and materials}

The datasets used and/or analyzed during the current study are available from the corresponding author on reasonable request.

\section{Ethics approval and consent to participate}

The animal care and all procedures were in accordance with the National Institutes of Health Guide for Care and Use of Laboratory Animals.

\section{Competing interests}

The authors declare no conflict of interests.

\section{Author details}

${ }^{1}$ College of Food Sciences and Pharmaceutical Engineering, Zaozhuang University, Zaozhuang 277160, China. ${ }^{2}$ Shandong Academy of Chinese Medicine, Jinan 250014, China. ${ }^{3}$ College of pharmacy, Shandong University of Traditional Chinese Medicine, Jinan 250355, China. ${ }^{4}$ School of Chinese Materia Medica, Beijing University of Traditional Chinese Medicine, Beijing 100029, China.

Received: 27 April 2020 Accepted: 7 August 2020

Published online: 28 August 2020

\section{References}

Cantelli-Forti G, Maffei F, Hrelia P, Bugamelli F, Bernardi M, D'Intino P, Maranesi M, Raggi MA. Interaction of licorice on glycyrrhizin pharmacokinetics. Environ Health Perspect. 1994;102(Suppl. 9):65-8.

Chen JH, Wang FM, Liu J, Lee FSC, Wang XR, Yang HH. Analysis of alkaloids in Coptis chinensis Franch by accelerated solvent extraction combined with ultra performance liquid chromatographic analysis with photodiode array and tandem mass spectrometry detections. Anal Chim Acta. 2008;613(2):184-95.

Dai LJ, Zhang H, Li MY, Xi K, Guo ZJ. Comparative study of three different herbs extracted by semi-bionic enzyme extraction. Northwest Pharm J. 2014;29(4): $331-5$

Dong SQ, Fan HR, Li QS, Wei GL, Liu WH, Si DY. In vivo metabolic pathway of liquiritin in rats. Chin Tradit Herbal Drugs. 2014;45(17):2499-505.

Fang SQ, Qu QY, Zheng YF, Zhong HH, Shan CX, Wang F, Li CY, Peng GP. Structural characterization and identification of flavonoid aglycones in three glycyrrhiza species by liquid chromatography with photodiode array detection and quadrupole time-of-flight mass spectrometry. J Sep Sci. 2016; 39(11):2068-78.

Fu Y, Luo J, Qin J, Yang M. Screening techniques for the identification of bioactive compounds in natural products. J Pharmaceut Biomed. 2019: 168189-200.

Gao S, Liu J, Wang M, Liu Y, Meng X, Zhang T, Qi Y, Zhang B, Liu H, Sun X, Xiao $P$. Exploring on the bioactive markers of Codonopsis Radix by correlation analysis between chemical constituents and pharmacological effects. J Ethnopharmacol. 2019:23631-41.

Han J, Ye M, Xu M, Sun JH, Wang BR, Guo DA. Characterization of flavonoids in the traditional Chinese herbal medicine-Huangqin by liquid chromatography coupled with electrospray ionization mass spectrometry. J Chromatogr B. 2007:848355-62.

Huang X, Kong L, Li X, Chen X, Guo M, Zou H. Strategy for analysis and screening of bioactive compounds in traditional Chinese medicines. J Chromatogr B. 2004:812(1-2):71-84.

Li C, Zhang WY, Yu Y, Cheng CS, Han JY, Yao XS, Zhou H. Discovery of the mechanisms and major bioactive compounds responsible for the protective effects of Gualou Xiebai Decoction on coronary heart disease by network pharmacology analysis. Phytomedicine. 2019:56261-8.

Li Y. Comparative study of Phellodendron Chinense Schneid. and Coptis Chinensis Franch. Haerbin: Heilongjiang University of Chinese Medicine; 2013.

Liu DW, Yan GL, Fang Y, Liu SM. Utility of UPLC-ESI-TOF/MS for rapid analysis of the constituents in Scutellariae Radix. Infor Tradit Chin Med. 2012;29(4):20-4.

Miao WJ, Hu Y, Jia L, Zhang CX, Yang WZ, Zhang P, Guo DA. Profiling and identification of chemical components of Shenshao Tablet and its absorbed components in rats by comprehensive HPLC/DAD/ESI-MSn analysis. Chin J Nat Med. 2018;16(10):791-800.

Su X, Kong L, Li X, Chen X, Guo M, Zou H. Screening and analysis of bioactive compounds with biofingerprinting chromatogram analysis of traditional Chinese medicines targeting DNA by microdialysis/HPLC. J Chromatogr A. 2005;1076(1-2):118-26. 
Wang HY, Yan GL, Zhang AH, Li Y, Wang YY, Sun H, Wu XH, Wang XJ. Rapid discovery and global characterization of chemical constituents and rats metabolites of Phellodendri amurensis cortex by ultra-performance liquid chromatography-electrospray ionization/quadrupole-time-of-flight mass spectrometry coupled with pattern recognition approach. Analyst. 2013b; 138(11):3303-12.

Wang HZ, Yu CH, Gao J, Zhao GR. Enzymatic extraction of baicalein and wogonin from Radix Scutellariae. Chin Med Mat. 2007;30(7):851-4.

Wang JL. Study on active substance in vivo origin and the pharmacokinetics of Erhuang decoction $\leq 1000 \mathrm{Da}$ extract prepared by "semi bionic enzyme extraction". Jinan: Shandong University of Traditional Chinese Medicine; 2014.

Wang JL, Sun XM, Wang YZ, Zhou Y, Zhang C. Comparative study on extracts of Erhuang decoction by three methods. Liaoning J Tradit Chin Med. 2013a; 40(7):1430-2.

Wang JL, Sun XM, Zhang ZW, Huang YL, Jiang RY, Zhou Y. Technique optimization of semi-bionic extraction for Erhuang decoction. World J Integr Tradit West Med. 2011;6(1):56-9.

Wang JL, Zheng DD, Sun XM, Wang YZ, Zhang C, Yu DR, Wang ZY. Ownership study of HPLC fingerprints of Erhuang decoction extracts with SBE method. Chin J Pharm Anal. 2016c;36(9):1563-70.

Wang JL, Zheng DD, Wang YZ, Zhang C, Sun XM. Pharmacokinetics study of Erhuang decoction extracts in rats by HPLC-MS/ MS. J Chromatogr B. 2017; 1059:35-42.

Wang L, Liu S, Xing J, Liu Z, Song F. Characterization of interaction property of multi-components in Gardenia jasminoides with aldose reductase by microdialysis combined with liquid chromatography coupled to mass spectrometry. Rapid Commun Mass Spectrom. 2016a;30(Suppl. 1):87-94.

Wang R, Wu G, Du L, Shao J, Liu F, Yang Z, Liu D, Wei Y. Semi-bionic extraction of compound turmeric protects against dextran sulfate sodium-induced acute enteritis in rats. J Ethnopharmacol. 2016b:190288-300.

Wang X, Sun W, Sun H, Lv H, Wu Z, Wang P, Liu L, Cao H. Analysis of the constituents in the rat plasma after oral administration of Yin Chen Hao Tang by UPLC/Q-TOF-MS/MS. J Pharmaceut Biom. 2008;46(3):477-90.

Wu Q. Golden mirror of medicine (photocopy). Beijing: People's Medical Publishing House; 1957.

Xiao HT, Qi XL, Liang Y, Lin CY, Wang X, Guan ZZ, Hao XY. Membrane permeability-guided identification of neuroprotective components from Polygonum cuspidatun. Pharm Biol. 2014;52(3):356-61.

Xu N, Shi HY. Analysis to the meaning of semi-bionic extraction method. J Liaoning Univ Tradit Chin Med. 2011;13(12):38-40.

Yamamura Y, Santa T, Kotaki H, Uchino K, Sawada Y, Iga T. Administration-route dependency of absorption of glycyrrhizin in rats: intraperitoneal administration dramatically enhanced bioavailability. Biol Pharm Bull. 1995; 18(2):337-41.

Yuan J, Chen Y, Liang J, Wang CZ, Liu X, Yan Z, Tang Y, Li J, Yuan CS. Component analysis and target cell-based neuroactivity screening of Panax ginseng by ultraperformance liquid chromatography coupled with quadrupole-time-of-flight mass spectrometry. J Chromatogr B. 2016;1038:1-11.

Zhang H, Zhao TT, Dai LJ, You XJ, Guo ZJ. Semi-bionic enzyme extraction method of glycyrrhizic acid in Glycyrrhizae Radix et Rhizoma. Chin J Mod Appl Pharm. 2013;30(9):969-72.

Zhang HY, Hu CX, Liu CP, Li HF, Wang JS, Yuan KL, Tang JW, Xu GW. Screening and analysis of bioactive compounds in traditional Chinese medicines using cell extract and gas chromatography-mass spectrometry. J Pharmaceut Biomed. 2007a;43(1):151-7.

Zhang L, Zhang RW, Li Q. Development of the fingerprints for the quality evaluation of Scutellariae Radix by HPLC-DAD and LC-MS-MS. Chromatographia. 2007b:6613-20.

Zhao YM, Liu SX, Zhang CX, Liu DL, Zhang TJ. Analysis on chemical constituents from Glycyrrhizae Radix et Rhizoma by HPLC-Q-TOF-MS. Chin Tradit Herbal Drugs. 2016:47(12):2061-8

Zuo R, Wang HJ, Si N, Zhao HY, Yang J, Bian BL. LC-FT-ICR-MS analysis of the prototypes and metabolites in rat plasma after administration of Huang-LianJie-Du decoction. Acta Pharm Sin. 2014;49(2):237-43.

\section{Publisher's Note}

Springer Nature remains neutral with regard to jurisdictional claims in published maps and institutional affiliations.

\section{Submit your manuscript to a SpringerOpen ${ }^{\circ}$ journal and benefit from:}

- Convenient online submission

- Rigorous peer review

- Open access: articles freely available online

High visibility within the field

- Retaining the copyright to your article

Submit your next manuscript at $\boldsymbol{\nabla}$ springeropen.com 